\title{
Evaluación y relevancia del magnesio sérico en pacientes adultos incidentes a la sala de emergencia de un hospital general de Lima, Perú
}

\begin{abstract}
Evaluation and relevance of serum magnesium values in adult patients attending the emergency room of a general hospital in Lima, Peru
\end{abstract}

Javier Antonio Cieza Zevallos ${ }^{1, a ; 2, b}$, Camilla Sofía Agurto García ${ }^{1, c}$, Diego Danilo Gayoso Liviacec,

\section{RESUMEN}

Objetivos: Describir la frecuencia y características de las alteraciones del magnesio sérico en pacientes que acuden a la sala de emergencia de un hospital nacional de Lima del Ministerio de Salud del Perú. Material y métodos: Estudio descriptivo con muestreo accidental de 180 pacientes adultos admitidos para observación intrahospitalaria u hospitalización en el área de medicina. Se registraron sus datos al momento de la admisión. Se realizó un análisis univariado para definir las variables asociadas a las alteraciones del magnesio y finalmente se llevó a cabo un análisis de regresión logística binaria. El análisis estadístico se realizó utilizando el paquete SPSS V18.0. Resultados: Hipomagnesemia estuvo presente en el 16,4\% de la muestra estudiada y la hipermagnesemia en el $18 \%$. Se encontró relación entre algunas de las variables estudiadas y los diferentes niveles de magnesio sérico $(\mathrm{p}<0,05)$. Conclusiones: Las alteraciones séricas del magnesio no son infrecuentes y es importante incorporar formas de medir la situación del magnesio corporal en forma más precisa, además considerar su asociación con la función renal en pacientes con disfunción severa.

PALABRAS CLAVE: Magnesio, hospitalización, electrolitos. (Fuente: DeCS BIREME).

\section{SUMMARY}

Objectives: To describe the frequency and features of serum magnesium derangements in patients attending the emergency room of a general hospital in Lima, Peru. Methods: A descriptive study was carried-out including 180 adult patients admitted to the observation unit of the emergency room selected by accidental sampling. Univariate and binary logistic regression were performed using the SPSS V18.0 software. Results: hypomagnesemia was found in $16.4 \%$ and hypermagnesemia in $18 \%$. An association between certain variables and serum levels of magnesium was found ( $\mathrm{p}<0.05)$. Conclusions: Serum magnesium derangements are frequent; it is important to evaluate these derangements with renal function as well as to measure magnesium body levels more accurately.

KEYWORDS: Magnesium, hospitalization, electrolytes. (Source: MeSH NLM).

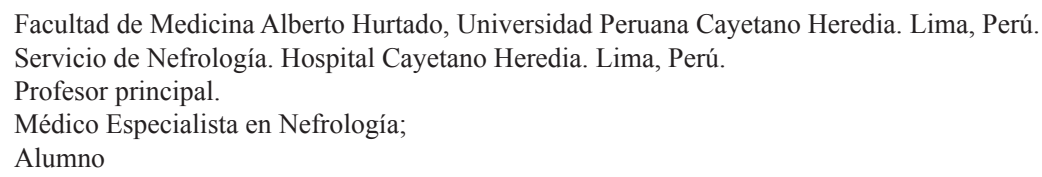




\section{INTRODUCCIÓN}

El magnesio es un catión divalente muy relevante en nuestra vida y fundamental para nuestra salud. Es el cuarto catión más abundante en el organismo y el segundo catión más abundante en el compartimiento intracelular. Cerca del $99 \%$ del magnesio total se localiza en el hueso, músculos y tejidos blandos no musculares. El magnesio extracelular supone cerca del $1 \%$ y se encuentra fundamentalmente en el suero y en los hematíes (1); su importancia puede deducirse de sus funciones y roles, como el ser un elemento fundamental para mantener la estabilidad de las membranas celulares o ser indispensable para la síntesis de numerosas enzimas y compuestos ricos en energía. En la fisiología humana, contribuye a la excitabilidad neuronal y muscular, a la estabilidad de los eritrocitos, al crecimiento y maduración óseo, a la motilidad de los espermatozoides, a la síntesis del surfactante pulmonar y en el corazón es un protector cardíaco anti-hipoxémico y anti-isquémico, protector de los vasos sanguíneos y funcionalmente actúa como un vasodilatador. Hay muchas otras funciones del magnesio que nos han permitido comprender en las últimas décadas su importancia en nuestra salud y la relevancia de sus alteraciones en las enfermedades.

Sin embargo, en países en vías de desarrollo como el nuestro, su significado clínico aún no es claramente entendido por los médicos, probablemente porque se conoce poco su relación con las patologías que más frecuentemente son atendidas, así también como se ignora la magnitud e importancia de sus alteraciones en poblaciones económicamente marginadas. Esta situación se torna más relevante cuando los servicios de salud con capacidad resolutiva de alta o mediana complejidad son ofertados a este tipo de poblaciones.

Cieza et al (2), han descrito que hasta $81,5 \%$ de los pacientes que acudieron al servicio de emergencia de un hospital general de Lima del Ministerio de Salud (MINSA) tuvieron hipoalbuminemia, una forma de traducir la desnutrición, y Lluncor et al (3), encontraron que existe una clara asociación entre la incidencia de injuria renal aguda y la vulnerabilidad biológica de la persona. Estas observaciones conllevan la necesidad de estudiar con mayor profundidad ciertos problemas del medio interno poco conocidos en nuestras poblaciones de pacientes, como las alteraciones del ion divalente, en especial el magnesio, que mayormente son subestimados y que pueden tener relevancia clínica y terapéutica.
Tener identificadas las alteraciones del magnesio en poblaciones vulnerables, es relevante porque permitirá al médico una mejor comprensión de ciertas manifestaciones de la enfermedad, que muchas veces no son explicadas claramente en ausencia de esta información. También es importante investigar las alteraciones del magnesio y su asociación con otros elementos del medio interno que permitan minimizar intercurrencias dependientes de estas alteraciones que pueden ser graves y mortales. El estudio es oportuno dado que esta información en poblaciones como las nuestras es escasa y las conductas médicas al respecto están sustentadas en referencias provenientes de países desarrollados, que podrían diferir de la de los países en vías de desarrollo.

En consecuencia, el estudio tuvo como objetivo describir las alteraciones del magnesio sérico en una población que acudió a solicitar servicios de emergencia en un hospital de salud de Lima Metropolitana, Perú, ubicado en el Cono Norte de la ciudad que atiende fundamentalmente pacientes económicamente vulnerables, afiliados al Seguro Integral de Salud (SIS). Además, analizar las variables personales, clínicas y del medio interno más relevantes asociadas a estos disturbios.

\section{MATERIAL Y MÉTODOS}

Estudio descriptivo con muestreo accidental. Se incluyeron pacientes que tuvieron dosaje de magnesio sérico al momento de ser admitidos a la sala de emergencia del Hospital Cayetano Heredia ubicado en el Cono Norte de Lima Metropolitana, Perú, en el mes de setiembre del 2016.

Se calculó un tamaño muestral en función de una población mensual promedio de 180 pacientes adultos que son admitidos para observación intrahospitalaria u hospitalización en el área de medicina; aceptando una probabilidad teórica de encontrar alteraciones del magnesio sérico del $50 \%$ con un error probabilístico del 5\%, el tamaño de la muestra calculada fue de 123 pacientes.

Ingresaron al estudio todos los pacientes a quienes se solicitó dosaje de magnesio sérico según criterio del médico. El muestreo fue accidental hasta completar el tamaño calculado y se inició la recolección de información desde el 1 de setiembre del 2016 hasta completar el tamaño muestral calculado. 
Se registraron los datos al momento de la admisión relativos a sexo, edad, peso, talla, presión arterial, frecuencia cardíaca, frecuencia respiratoria, creatinina y urea séricas, recuento de leucocitos y linfocitos, electrolitos séricos (sodio, potasio y cloro), calcio total y calcio ionizado, fósforo sérico, albúmina sérica y gasometría arterial. Los datos clínicos se registraron como variables categóricas la existencia o no de sepsis, disfunción cardíaca, respiratoria, compromiso neurológico o disfunción hepática de cualquier tipo. También se registró la coexistencia de diarrea, enfermedad inmunológica y cáncer. La gravedad se registró considerando tres categorías: muy grave (paciente con inestabilidad hemodinámica o estado de shock; categoría 3), grave (paciente sin inestabilidad hemodinámica, pero con compromiso funcional de más de dos sistemas; categoría 2) y paciente relativamente estable (paciente sin inestabilidad hemodinámica y con uno o dos sistemas comprometidos funcionalmente; categoría 1).

Se consideró hipomagnesemia, magnesio sérico $<1,7 \mathrm{mg} / \mathrm{dl}$; hipermagnesemia, magnesio sérico $>2,2$ $\mathrm{mg} / \mathrm{dl}$ y magnesio normal valores entre 1,7 y $2,2 \mathrm{mg} / \mathrm{dl}$. Se determinó la frecuencia de hipo o hipermagnesemia, y posteriormente se realizó un análisis univariado para definir las variables asociadas a las alteraciones del magnesio (hipo, normo e hipermagnesemia) y mediante análisis de regresión logística binaria, las variables asociadas a hipo o hipermagnesemia en relación con los pacientes con magnesio normal. Se aceptó como significancia estadística un $p<0,05$. El análisis estadístico se realizó utilizando el paquete SPSS V18.0.
El estudio fue revisado, aprobado y autorizado por el Departamento de Emergencia y por el Comité de Ética del Hospital Cayetano Heredia. Los datos de cada paciente fueron y son guardados confidencialmente y solamente fueron utilizados para este estudio.

\section{RESULTADOS}

Se incluyeron para el estudio 122 pacientes, un paciente fue excluido por ser menor de 18 años. El $52,8 \%$ de los pacientes fueron varones y el $47,2 \%$, mujeres. La edad promedio fue 56,63 $\pm 22,03$ años, la talla promedio fue $1,58 \pm 0,07 \mathrm{~m}$ y el peso promedio $61,15 \pm 0,07 \mathrm{~kg}$. El índice de masa corporal (IMC) fue $24,10 \pm 4,13 \mathrm{~kg} / \mathrm{m} 2: 6(5,7 \%)$ pacientes tuvieron IMC $<18,5,57(53,8 \%)$ IMC entre 18,5 y 24; $35(33 \%)$ IMC entre 25 y 29 , y $8(7,5 \%)$ IMC $\geq 30$.

En la tabla 1 se muestra los hallazgos de laboratorio de la población estudiada. La frecuencia de alteraciones de la gasometría arterial y los diversos sistemas orgánicos disfuncionales evaluados se muestran en la tabla 2. Solamente fue identificado un paciente con cáncer en la población estudiada. Respecto a la gravedad de los pacientes, $19(15,6 \%)$ pacientes calificaron en la categoría 1; $56(45,9 \%)$ en la categoría 2 y $47(38,5 \%)$ en la categoría 3.

Ochenta(64,6\%)pacientes tuvieronmagnesio sérico en valores normales, se encontró hipomagnesemia en $20(16,4 \%)$ pacientes e hipermagnesemia en 22 (18\%).

El análisis de correlación de Pearson entre los valores del magnesio sérico solo mostró correlación

Tabla 1. Hallazgos de laboratorio de la población estudiada.

\begin{tabular}{lccc}
\hline Variable & n & Promedio \pm DS & Rango Normal \\
\hline Magnesio sérico $(\mathrm{mg} / \mathrm{dl})$ & 122 & $2,04 \pm 0,57$ & $1,7-2,2$ \\
Creatinina sérica $(\mathrm{mg} / \mathrm{dl})$ & 122 & $2,45 \pm 3,59$ & $0,6-1,2$ \\
Urea sérica $(\mathrm{mg} / \mathrm{dl})$ & 106 & $75,37 \pm 58,10$ & $20-40$ \\
Albúmina sérica $(\mathrm{g} / \mathrm{dl})$ & 50 & $2,97 \pm 0,71$ & $3,40-5,40$ \\
Calcio total $(\mathrm{mg} / \mathrm{dl})$ & 88 & $8,35 \pm 1,02$ & $8,50-10,20$ \\
Calcio ionizado $(\mathrm{mmol} / \mathrm{l})$ & 80 & $1,18 \pm 0,15$ & $1,20-1,40$ \\
Fósforo sérico $(\mathrm{mg} / \mathrm{dl})$ & 95 & $4,53 \pm 1,87$ & $2,40-4,10$ \\
Sodio sérico $(\mathrm{mEq} / \mathrm{l})$ & 122 & $138,89 \pm 7,52$ & $135-145$ \\
Potasio sérico $(\mathrm{mEq} / \mathrm{l})$ & 122 & $4,27 \pm 0,88$ & $3,5-5,0$ \\
Cloro sérico $(\mathrm{mEq} / \mathrm{l})$ & 122 & $101 \pm 7,17$ & $96-106$ \\
Recuento leucocitos $\left.(\mathrm{x} \mathrm{mm})^{3}\right)$ & 119 & $11906 \pm 9102$ & $4500-11000$ \\
Recuento linfocitos $\left.(\mathrm{x} \mathrm{mm})^{3}\right)$ & 119 & $1299 \pm 814$ & $1300-4000$ \\
\hline
\end{tabular}


Tabla 2. Hallazgos de la gasometría arterial y compromiso clínico de la población estudiada.

\begin{tabular}{lcr}
\hline Variable & $\mathbf{n}$ & $\mathbf{\%}$ \\
\hline Gasometría Normal & $18 / 116$ & 15,5 \\
Alcalosis Respiratoria & $86 / 116$ & 74,1 \\
Acidosis Metabólica & $26 / 116$ & 22,4 \\
Alcalosis Metabólica & $18 / 116$ & 15,5 \\
Acidosis Respiratoria & $7 / 116$ & 6,0 \\
Alcalosis Respiratoria y Acidosis Metabólica & $20 / 116$ & 17,3 \\
Estado de sepsis & $51 / 122$ & 41,8 \\
Insuficiencia Respiratoria & $61 / 122$ & 50,0 \\
Insuficiencia Cardíaca & $23 / 122$ & 18,9 \\
Disfunción neurológica & $35 / 122$ & 28,7 \\
Insuficiencia hepática & $5 / 122$ & 4,1 \\
Diarrea aguda o crónica & $5 / 122$ & 4,1 \\
Enfermedad inmunológica & $7 / 122$ & 5,7 \\
\hline
\end{tabular}

Tabla 3. Regresión lineal multivariado en relación al magnesio sérico.

\begin{tabular}{lcccc}
\hline \multicolumn{2}{l}{ Valor del modelo: constante $\boldsymbol{\beta} \mathbf{1 6 , 2 0 7} \pm \mathbf{5 , 9 6 8 ;} \boldsymbol{p}=\mathbf{0 , 0 0 8}$} & & \\
Variables & Coeficiente $\boldsymbol{\beta} \pm \mathbf{E E}$ & $\mathbf{C o e f i c i e n t e}$ tipificado & $\mathbf{t}$ & $\boldsymbol{p}$ \\
\hline Frecuencia cardíaca & $0,007 \pm 0,002$ & 0,264 & $\mathbf{3 , 0 5 9}$ & $\mathbf{0 , 0 0 3}$ \\
Creatinina & $0,019 \pm 0,014$ & 0,125 & 1,442 & 0,152 \\
$\mathrm{pH}$ arterial & $-1,981 \pm 0,815$ & $-0,263$ & $\mathbf{- 2 , 4 3 0}$ & $\mathbf{0 , 0 1 7}$ \\
$\mathrm{HCO}_{3}^{-}$ & $-0,006 \pm 0,011$ & $-0,061$ & $-0,602$ & 0,548 \\
\hline
\end{tabular}

Tabla 4. Variables cualitativas asociadas estadísticamente con alteraciones del Magnesio sérico.

\begin{tabular}{|c|c|c|c|c|}
\hline Variable & $\begin{array}{c}\text { Hipomagnesemia } \\
\text { n }(\%)\end{array}$ & $\begin{array}{c}\text { Magnesio normal } \\
\text { n (\%) }\end{array}$ & $\begin{array}{c}\text { Hipermagnesemia } \\
\text { n (\%) }\end{array}$ & $\mathbf{p}$ \\
\hline \multicolumn{5}{|l|}{ Potasio sérico } \\
\hline Bajo & $7(36,8 \%)$ & $11(57,9 \%)$ & $1(5,3 \%)$ & \multirow{3}{*}{0,041} \\
\hline Normal & $11(12,1 \%)$ & $63(69,2 \%)$ & $17(18,7 \%)$ & \\
\hline Alto & $2(16,7 \%)$ & $6(50,0 \%)$ & $4(33,3 \%)$ & \\
\hline \multicolumn{5}{|c|}{ Acidosis Metabólica } \\
\hline Presente & $1(3,8 \%)$ & $15(57,7 \%)$ & $10(38,5 \%)$ & \multirow[t]{2}{*}{0,006} \\
\hline Ausente & $18(20,0 \%)$ & $60(66,7 \%)$ & $12(13,3 \%)$ & \\
\hline \multicolumn{5}{|c|}{ Alcalosis Metabólica } \\
\hline Presente & $11(61,1 \%)$ & $7(38,9 \%)$ & $0(0,0 \%)$ & \multirow[t]{2}{*}{0,000} \\
\hline Ausente & $8(8,2 \%)$ & $68(69,4 \%)$ & $22(22,4 \%)$ & \\
\hline \multicolumn{5}{|c|}{ Alcalosis Respiratoria } \\
\hline Presente & $16(18,6 \%)$ & $59(68,6 \%)$ & $11(12,8 \%)$ & \multirow[t]{2}{*}{0,014} \\
\hline Ausente & $3(10,0 \%)$ & $16(53,3 \%)$ & $11(36,7 \%)$ & \\
\hline \multicolumn{5}{|c|}{ Alcalosis Respiratoria y } \\
\hline Acidosis Meta & & & & \multirow{3}{*}{0,008} \\
\hline Presente & $0(0,0 \%)$ & $12(60,0 \%)$ & $8(40,0 \%)$ & \\
\hline Ausente & $19(19,8 \%)$ & $63(65,6 \%)$ & $14(14,6 \%)$ & \\
\hline \multicolumn{5}{|l|}{ Sepsis } \\
\hline Presente & $12(23,5 \%)$ & $25(49,0 \%)$ & $14(27,5 \%)$ & \multirow[t]{2}{*}{0,005} \\
\hline Ausente & $8(11,3 \%)$ & $55(77,5 \%)$ & $8(11,3 \%)$ & \\
\hline \multicolumn{5}{|c|}{ Insuficiencia Respiratoria } \\
\hline Presente & $12(19,7 \%)$ & $33(54,1 \%)$ & $16(26,2 \%)$ & \multirow[t]{2}{*}{0,020} \\
\hline Ausente & $8(13,1 \%)$ & $47(77,0 \%)$ & $6(9,8 \%)$ & \\
\hline
\end{tabular}


estadísticamente significativa con la frecuencia cardíaca $(\mathrm{r}=0,302 ; \mathrm{p}=0,001) ;$ la creatinina sérica $(\mathrm{r}=0,186 ; \mathrm{p}=0,04)$ y en la gasometría de sangre arterial con el $\mathrm{pH}(\mathrm{r}=-0,351 ; \mathrm{p}=0,000)$ y el $\mathrm{HCO}_{3}^{-}(\mathrm{r}=0,206$; $\mathrm{p}=0,026$ ). Sin embargo, el análisis de regresión lineal multivariado, incluyendo las variables antes mencionadas, mostraron un modelo relacionado fundamentalmente al $\mathrm{pH}$ de sangre arterial y a la frecuencia cardíaca (Tabla 3).

No se encontró asociación entre las alteraciones del magnesio con la edad categorizada en grupos etarios $(\mathrm{p}=0,973)$, ni con el IMC $(\mathrm{p}=0,231)$, ni con la gravedad de los pacientes $(\mathrm{p}=0,132)$, ni con el sexo $(\mathrm{p}=0,604)$. Las variables categóricas que alcanzaron asociación estadística con las alteraciones del magnesio, se muestran en la tabla 4. Las alteraciones del fósforo sérico $(\mathrm{p}=0,177)$, calcio ionizado $(\mathrm{p}=0,233)$, calcio total $(\mathrm{p}=0,876)$, acidosis respiratoria $(\mathrm{p}=0,924)$, insuficiencia cardíaca $(\mathrm{p}=0,514)$ y disfunción neurológica $(p=0,389)$ no mostraron significancia estadística. La albúmina sérica solamente mostró asociación significativa con la hipomagnesemia $(\mathrm{p}=0,019)$, cuando ésta fue menor de $2,5 \mathrm{~g} / \mathrm{dl}$, en tanto la hipermagnesemia no mostró asociación significativa con los valores de albúmina sérica.

El análisis de regresión logística multinominal realizado con las variables que tuvieron significancia estadística según la tabla 4, mostró como modelo final relacionado con las alteraciones del magnesio a la coexistencia de alcalosis metabólica, alcalosis respiratoria, la presencia del disturbio mixto de alcalosis respiratoria y acidosis metabólica y la presencia de sepsis.

Se encontró diferencia significativa entre los pacientes con hipomagnesemia y magnesio normal en el calcio ionizado (Gráfico1), la albúmina sérica, el recuento leucocitario y la relación urea/ creatinina (Tabla 5). En las variables categóricas sólo la sepsis y la alcalosis metabólica estuvieron asociadas a la hipomagnesemia. Pacientes con sepsis e hipomagnesemia fueron $32,4 \%$, mientras pacientes sin sepsis e hipomagnesemia fueron $12,7 \%,(p=0,017)$; mientras que, pacientes con alcalosis metabólica e hipomagnesemia fueron $61,1 \%$ y pacientes sin alcalosis metabólica e hipomagnesemia fueron 10,5\% $(\mathrm{p}=0,000)$.

En relación con la hipermagnesemia se encontró diferencias estadísticamente significativas con los pacientes con magnesio normal en la urea sérica (hipermagnesemia 110,65 $\pm 86,55 \mathrm{mg} / \mathrm{dl}$ y normomagnesemia $70,11 \pm 77,23 \mathrm{mg} / \mathrm{dl}, \mathrm{p}=0,036$ ) y el recuento leucocitario (hipermagnesemia 14499 $\pm 6267 \times \mathrm{mm}^{3}$ y normomagnesemia $10183 \pm 5$ $\left.448 \times \mathrm{mm}^{3}, \mathrm{p}=0,002\right)$, y en las variables categóricas sepsis, presencia de disfunción respiratoria, acidosis metabólica, alcalosis respiratoria y coexistencia de alcalosis respiratoria con acidosis metabólica se encontró asociación con hipermagnesemia (Tabla 6).

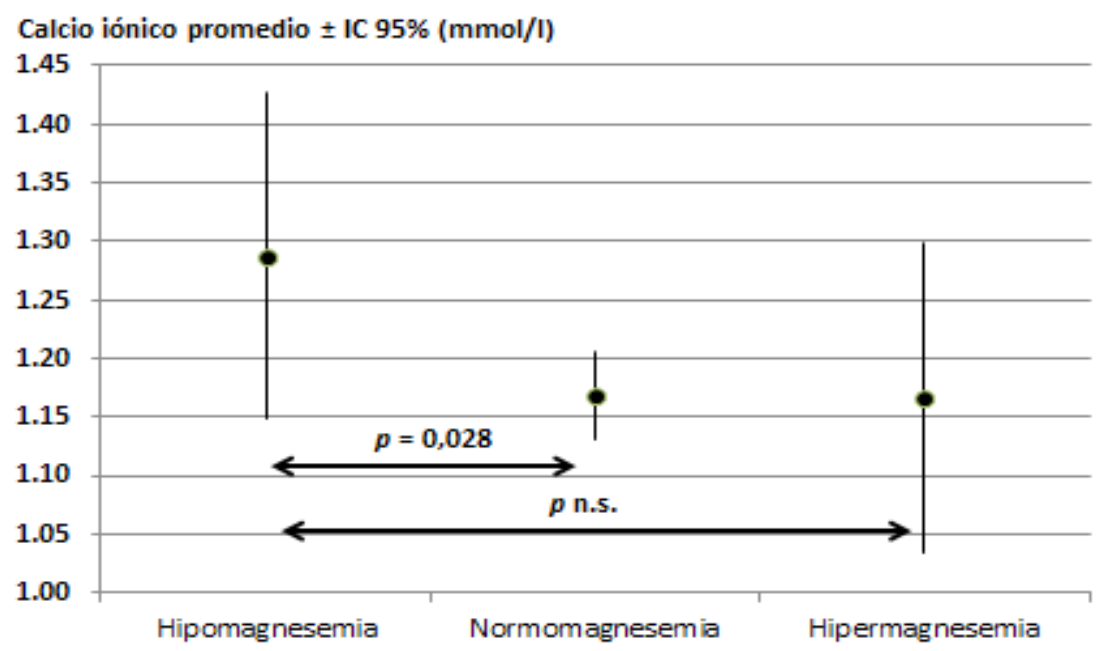

Gráfico 1. Relación entre los niveles del magnesio sérico y el calcio ionizado. 
Tabla 5. Comparación de las medias de las variables cuantitativas que mostraron diferencia significativa en relación a la presencia de hipomagnesemia.

\begin{tabular}{lccc}
\hline Variable & Hipomagnesemia & Normomagnesemia & $\boldsymbol{p}$ \\
\hline Albúmina sérica $(\mathrm{g} / \mathrm{dl})$ & $2,42 \pm 0,54$ & $3,08 \pm 0,58$ & 0,004 \\
Calcio ionizado $(\mathrm{mmol} / \mathrm{l})$ & $1,29 \pm 0,24$ & $1,17 \pm 0,14$ & 0,028 \\
Recuento leucocitario $\left({ }^{*} \mathrm{~mm}^{3}\right)$ & $15685 \pm 17820$ & $10183 \pm 5448$ & 0,021 \\
Relación urea/creatinina & $60,20 \pm 41,27$ & $46,03 \pm 24,03$ & 0,047 \\
\hline
\end{tabular}

Tabla 6. Comparación de las variables categóricas asociadas a la presencia de hipermagnesemia en relación a la normomagnesemia.

\begin{tabular}{lccc}
\hline Variable & $\begin{array}{c}\text { Hipermagnesemia } \\
\mathbf{n ~ ( \% )}\end{array}$ & $\begin{array}{c}\text { Normomagnesemia } \\
\mathbf{n}(\mathbf{\%})\end{array}$ & $\boldsymbol{p}$ \\
\hline Sepsis & $14(35,9 \%)$ & $8(12,7 \%)$ & 0,006 \\
Insuficiencia Respiratoria & $16(32,7 \%)$ & $6(11,3 \%)$ & 0,009 \\
Alcalosis respiratoria & $11(15,7 \%)$ & $11(40,7 \%)$ & 0,008 \\
Acidosis Metabólica & $10(40 \%)$ & $12(16,7 \%)$ & 0,016 \\
Alcalosis Respiratoria y Acidosis Metabólica & $8(40 \%)$ & $14(18,2 \%)$ & 0,038 \\
\hline
\end{tabular}

\section{DISCUSIÓN}

El estudio tuvo como objetivo principal describir la frecuencia y características de las alteraciones del magnesio sérico en una población de pacientes que acuden a una sala de emergencia de medicina de un hospital general de Lima del Ministerio de Salud del Perú. Aceptando que las características de la muestra no es la ideal pues fue tomada en forma accidental y por conveniencia en función de que no a todos los pacientes que acuden a esta emergencia se les solicita control de magnesio sérico, esta observación es una primera aproximación formal en la descripción de las alteraciones de este catión divalente tan importante para nuestra salud a nivel local y probablemente a nivel regional.

A nivel de la literatura internacional la frecuencia de hipomagnesemia e hipermagnesemia en pacientes hospitalizados en general ha sido hallada en $11 \%$ y $9,3 \%$ respectivamente (4), pero en pacientes que ingresan a unidades de cuidados intensivos puede ser hasta 44\% (5), habiéndose descrito una mayor letalidad asociada a la hipomagnesemia (6).

Este estudio mostró que la hipomagnesemia estuvo presente en el 16,4\% de la muestra estudiada y la hipermagnesemia en el $18 \%$. Comparado con las cifras antes referidas, es importante resaltar la mayor frecuencia observada de alteraciones del magnesio sérico, hecho que puede explicarse por la mayor vulnerabilidad nutricional de los pacientes aquí estudiados: el $74 \%$ de los pacientes a quienes se les había solicitado albúmina sérica tuvieron valores menores de 3,4 g/dl (hipoalbuminemia), y 36\% tuvieron un valor inferior a $2,5 \mathrm{~g} / \mathrm{dl}$ (hipoalbuminemia severa).

En términos generales, los valores de magnesio se relacionaron directamente con la frecuencia cardíaca e inversamente con el estado de alcalemia. Estas alteraciones se asociaron esencialmente con el estado séptico cuando éste presenta alcalosis respiratoria sola o como trastorno mixto con acidosis metabólica. Como es sabido, la sepsis presenta alcalosis respiratoria y acidosis metabólica por sus efectos de estímulo de la frecuencia respiratoria y el estado catabólico de la persona, esto explicaría que el modelo final estuviera relacionado con la sepsis, el trastorno mixto de acidosis metabólica con alcalosis respiratoria y la alcalosis respiratoria sola. La alcalosis metabólica aquí observada, hipotéticamente ocurriría por contracción de la volemia cuando hay pérdida del agua corporal en personas desnutridas con pérdidas de la presión oncótica (la mayoría de los pacientes tuvieron hipoalbuminemia y un porcentaje importante tuvieron hipoalbuminemia severa). Estos hallazgos se relacionan con lo encontrado en la literatura, que describe que los valores de magnesio disminuyen aproximadamente de 4 a $8 \%$ por cada 0,1 de aumento en el $\mathrm{pH}$ en los pacientes hospitalizados (7).

Cuando se compararon pacientes con hipomagnesemia y con hipermagnesemia con 
los pacientes con magnesio normal, un común denominador fue la asociación con la sepsis para ambos trastornos, con los disturbios de la gasometría sanguínea esperable y resaltando que solamente en la hipomagnesemia se apreció un recuento leucocitario mayor que en los pacientes con magnesio sérico normal. Desde la perspectiva puramente clínica, la otra diferencia hallada fue que la insuficiencia respiratoria estuvo asociada a hipermagnesemia. Esta situación podría corresponder a procesos dependientes de otras variables aquí no estudiadas o cuya presencia no alcanzó significancia propia por el número de observaciones realizadas, como la insuficiencia renal, que en el análisis simple de correlación de Pearson sí tuvo asociación positiva con el magnesio sérico, pero no fue parte del modelo final de regresión lineal múltiple. Es conocido que la insuficiencia renal se asocia a hipermagnesemia y a disfunción respiratoria en sus estados disfuncionales avanzados.

Una última observación importante que debe resaltarse es la relación de la hipomagnesemia con la hipercalcemia, no así de la hipermagnesemia con hipocalcemia. Esta situación es probable que dependa principalmente de las características de la población estudiada (desnutrición altamente prevalente) en donde solamente el extremo de la relación sea perceptible (la hipomagnesemia con calcio ionizado mayor que la de pacientes con valores normales de magnesio). Al respecto esta asociación puede verse diferente cuando el análisis del magnesio sérico se realiza con el calcio total como ha sido referida en la literatura (4), en donde la hipomagnesemia se asoció con hipocalcemia, situación propia de la vulnerabilidad biológica del paciente desnutrido y que no se refleja necesariamente cunado se mide el calcio ionizado.

En conclusión, este estudio ha mostrado que las alteraciones séricas del magnesio no son infrecuentes y su presencia es mayor que la descrita en otras publicaciones referentes a poblaciones equivalentes (esta población no incluyó mayormente pacientes con cáncer ni pacientes de cuidados críticos). También ha mostrado que en poblaciones vulnerables nutricionalmente, su asociación más relevante es aquella asociada a estados sépticos en quienes los disturbios del trastorno ácido base son muy frecuentes. Por estas razones sugerimos incorporar rutinariamente su evaluación y quizás incorporar formas de medir la situación del magnesio corporal en forma más precisa como su evaluación a través del contenido de magnesio intraeritrocitario, así también como considerar su asociación con la función renal en pacientes con disfunción severa.

\section{Declaración de financiamiento y de conflictos de intereses:}

El estudio fue financiado por los autores. Declaran no tener conflictos de intereses.

\section{Declaración de autoría:}

JACZ; CSAG y DDGL: Participaron en el diseño del estudio, interpretación de los resultados y aprobación de la versión final a ser publicada. CSAG y DDGL: recolección de los datos.

\section{Correspondencia:}

Javier Cieza

Correo electrónico: javier.cieza@upch.pe

\section{REFERENCIAS BIBLIOGRÁFICAS}

1. de Baaij J, Hoenderop J, Bindels R. Magnesium in man: Implications for health and disease. PhysiologicalReviews. 2015; 95(1):1-46.

2. Cieza J, Casillas A, Da Fieno A, Urtecho S. Asociación del nivel de albúmina sérica y alteraciones de los electrolitos, gases sanguíneos y compuestos nitrogenados en pacientes adultos incidentes del servicio de emergencia de un hospital general. Rev Med Hered. 2016; 27(4):223-229.

3. Lluncor J, Cruz-Encarnación M, Cieza J. Factores asociados a injuria renal aguda en pacientes incidentes de un hospital general de Lima- Perú. Rev Med Hered. 2015; 26(1):24-30.

4. Wong E, Rude R, Singer F, Shaw S. A high prevalence of hypomagnesemia and hypermagnesemia in hospitalized patients. Am J Clin Pathol. 1983; 79(3):348-52.

5. Reinhart R, Desbiens N. Hypomagnesemia in patients entering the ICU. Crit Care Med. 1985; 13(6):506-7.

6. Rubeiz G, Thill-Baharozian M, Hardie D, Carlson R. Association of Hypomagnesemia and mortality in acutely ill medical patients. Crit Care Med. 1993; 21(2):203-209.

7. Meza M. Disturbios del estado ácido-básico en el paciente crítico. Acta Médica Peruana. 2011; 28(1):46-55.

Recibido: $12 / 07 / 2017$

Aceptado: 06/03/2018 